\title{
Transsynaptic Activity-Dependent Regulation of Axon Branching and Neurotrophin Expression In Vivo
}

\author{
Anda-Alexandra Calinescu, ${ }^{1}$ Tiecheng Liu, ${ }^{1}$ Michael M. Wang, ${ }^{1,2,3}$ and Jimo Borjigin ${ }^{1}$ \\ ${ }^{1}$ Department of Molecular and Integrative Physiology and ${ }^{2}$ Department of Neurology, University of Michigan Medical School, and ${ }^{3}$ Veterans \\ Administration Ann Arbor Healthcare System, Ann Arbor, Michigan 48109-5622
}

The two major classes of activity-dependent neuroplasticity predict different consequences of activity alteration on circuit response. Hebbian plasticity (positive feedback) posits that alteration of neuronal activity causes a parallel response within a circuit. In contrast, homeostatic plasticity (negative feedback) predicts that altering neuronal activity results in compensatory responses within a circuit. The relative roles of these modes of plasticity in vivo are unclear, since neuronal circuits are difficult to manipulate in the intact organism. In this study, we tested the in vivo effects of activity deprivation in the superior cervical ganglion-pineal circuit of adult rats, which can be noninvasively silenced by exposing animals to constant light. We demonstrated that total deprivation of sympathetic activity markedly decreased the presence of axonal proteins in the pineal and reduced the density and thickness of sympathetic axonal arbors. In addition, we demonstrated that sympathetic inactivity eliminated pineal function and markedly decreased pineal expression of neurotrophins. Administration of $\beta$-adrenergic agonist restored the expression of presynaptic and postsynaptic proteins. Furthermore, compensatory axonal growth through collateral sprouting, normally seen following unilateral denervation of the pineal, was profoundly impaired in the absence of neural activity. Thus, these data suggest that sympathetic axonal terminals are maintained by neural activity that induces neurotrophins, which may act through a retrograde mechanism to preserve the integrity of axonal arbors via a positive feedback loop. Conversely, by using Hebbian-like neuroplasticity, silent yet intact circuits enter a hibernation mode marked by reduction of presynaptic axonal structures and dramatically reduced postsynaptic expression of neurotrophins.

\section{Introduction}

Activity-dependent changes in neural circuits have attracted widespread attention, largely due to their potential roles in learning, memory, and adaptation to disease states. Two dichotomous responses to changes in neuronal activity have been observed in neural circuits: (1) Hebbian responses typified by long-term potentiation (positive feedback), in which changes in network activity generate parallel changes in the response (Paulsen and Sejnowski, 2000); and (2) homeostatic neuroplasticity (negative feedback), in which a neural circuit is stabilized in response to activity changes (Nelson and Turrigiano, 2008). These principles have been extensively validated in in vitro and ex vivo models, but major questions remain unanswered. Most significantly, it remains uncertain whether in vivo neuroplasticity is governed by Hebbian or homeostatic principles. A significant obstacle to experimental testing in vivo is that manipulation of circuit activity in animals by non-

\footnotetext{
Received May 1, 2011; revised July 5, 2011; accepted July 12, 2011.

Author contributions: A.-A.C. and J.B. designed research; A.-A.C. and T.L. performed research; A.-A.C. analyzed data; A.-A.C., M.M.W., and J.B. wrote the paper.

This work was supported by NIH Grant NS057583 (J.B.) and Department of Defense Grants FA9550-08-0149 (J.B.) and FA9550-09-0352 (J.B.). We thank Dr. Peter Hitchcock for critically reading this manuscript, Dr. Roman Giger for helpful discussions, and Mr. Richard Chis for help with animal care.

The authors declare no competing financial interests.

Correspondence should be addressed to Jimo Borjigin, Department of Molecular and Integrative Physiology, University of Michigan Medical School, 1301 East Catherine Street, Ann Arbor, Ml 48109-5622. E-mail: borjigin@umich.edu.

DOI:10.1523/JNEUROSCI.2172-11.2011

Copyright $\odot 2011$ the authors $\quad 0270-6474 / 11 / 3112708-08 \$ 15.00 / 0$
}

pharmacological and noninvasive methods presents significant challenges (Nelson and Turrigiano, 2008).

The superior cervical ganglion (SCG)-pineal circuit (Bowers et al., 1984) offers an attractive in vivo model (Zigmond et al., 1985) to investigate activity-dependent neuroplasticity. The pineal gland releases melatonin in response to nightly norepinephrine secretion from clock-controlled SCG neurons (Borjigin et al., 1999). Norepinephrine activates $\beta 1$-adrenergic receptors on pinealocytes and increases melatonin production via arylalkylamine $N$-acetyltransferase (AANAT) activation (Borjigin et al., 1995; Klein et al., 1997). The hypothalamus-SCG-pineal circuit offers several unique advantages for the study of activity-dependent changes in neuronal networks: (1) it is a relatively simple, linear circuit; (2) the system can be rapidly and reproducibly silenced by exposure to light at night, which inhibits presynaptic norepinephrine release (Drijfhout et al., 1996) and activates AANAT degradation; and (3) melatonin production in constant light can be restored by adrenergic agonists (Huang et al., 2010). In the present study, our first objective was to test effects of prolonged inactivity on structural plasticity of axons in normal and injured pineal and to determine the ability of pharmacological activation of postsynaptic adrenergic receptors to alter structural plasticity of sympathetic axons in the absence of endogenous circuit activity.

Target-derived neurotrophins, including nerve growth factor (NGF) and neurotrophin 3 (NT3), promote survival and neurite extension of sympathetic neurons in vitro and ex vivo (LeviMontalcini and Angeletti, 1968; Campenot, 1977; Ruit et al., 1990; Hamburger, 1993). In adult animals, sympathetic fiber density cor- 
relates with NGF levels in target tissues (Korsching and Thoenen, 1983). Additionally, NGF promotes synaptic transmission between cultured sympathetic neurons and cardiomyocytes (Lockhart et al., 1997). It is not known, however, whether neuronal activity is continuously required for neurotrophin expression in target organs. Thus, our second objective was to test the effects of activity silencing on neurotrophin expression in normal and partially denervated pineal. Finally, we investigated whether adrenergic activation could restore neurotrophin expression under constant light.

Our data support the existence of Hebbian-like neuroplasticity within the SCG-pineal circuit: activity silencing suppresses presynaptic axonal growth and postsynaptic function and adrenergic receptor stimulation induces axonal growth of SCG neurons and postsynaptic function, both of which parallel changes in NGF expression in the pineal.

\section{Materials and Methods}

Animals. Adult male Fischer and Sprague Dawley rats were purchased from Harlan and housed in a light:dark (LD) cycle of 12:12 h (lights on at 6 A.M.) with food and water ad libitum for at least a week before experiments. After acclimating to our animal housing facility, animals were kept in chambers in LD or in constant light (LL; animals do not experience any darkness in LL) conditions for the times specified below, with light intensity between 250 and 450 lux at cage level. At selected time points, animals were killed by decapitation in the middle of the night (zeitgeber 18:00 h, or ZT18) under very dim red light for the LD animals and in the room light for the LL animals. At the time of death, animals were 10-15 weeks old (weight, $210-330 \mathrm{~g}$ ). Pineal glands were dissected and flash frozen in liquid nitrogen for subsequent analysis. All animal protocols were reviewed and approved by the Committee on Use and Care of Animals at the University of Michigan.

Surgery. Unilateral or bilateral surgical removal of the SCG was performed at 9 A.M. (ZT3), as described previously (Brammer et al., 1982). In brief, animals were anesthetized with isoflurane, a small incision of $\sim 1.5 \mathrm{~cm}$ was made at the level of the trachea, neck muscles (cleidocephalic, sternomastoid, sternothyroid, omohyoid) were separated, gently pulled to the side, and the common carotid artery was isolated. The internal and external carotid nerves (rostral) and the cervical sympathetic trunk (caudal), which connect the SCG to target tissues and spinal cord, respectively, were cut and the SCG removed. Incisions were sutured and animals were allowed to recover and then returned to their cages, where they were housed in either LD or LL conditions. At set times after surgery, animals were killed and pineal glands dissected and either flash-frozen in liquid nitrogen for subsequent Western blot analysis or fixed in 4\% paraformaldehyde, 5\% sucrose PBS for immunohistochemical analysis.

Antibodies and chemicals. For immunoblots, we used the following primary antibodies: anti- $\beta$-III tubulin (PRB-435P; Covance), antityrosine hydroxylase (TH; T-1299; Sigma), anti-AANAT generated in our laboratory (Huang et al., 2005), and anti-pan 14-3-3 (H-8) and anti-NGF (M-20) (Santa Cruz Biotechnology). Secondary antibodies used were anti-rabbit IgG, HRP-conjugated (AP307P; Millipore), and IRDye800CW-conjugated anti-mouse IgG (Rockland). Anti- $\beta$ III tubulin (PRB-435P; Covance) and anti-TH (AB1542; Millipore) were used for immunohistochemistry. Secondary antibodies were Alexa-Fluor 568 donkey anti-rabbit and Alexa-Fluor 488 donkey anti-mouse (Invitrogen). Normal donkey serum was purchased from Jackson ImmunoResearch and Hoechst 3332 was purchased from Invitrogen.

Isoproterenol $\mathrm{HCl}$ (I-104) was purchased from Research Biochemicals International. For the immunoblot analysis, protease inhibitor complete mini (Roche Diagnostics) and super signal west pico chemiluminescent substrate (Thermo Scientific) were used.

Tissue-Tek OCT compound was obtained from Electron Microscopy Sciences, RNeasy mini kit from Qiagen, Superscript II first strand synthesis kit for RT-PCR from Invitrogen, and Brilliant II SYBR Green QPCR master mix from Stratagene.

Western blot analysis. Frozen pineals were sonicated in lysis buffer (10 mм Tris $\mathrm{HCl}$, pH 7.5, with 1\% SDS, 0.5 mM EDTA, $0.1 \mathrm{~mm} \mathrm{Na}_{3} \mathrm{VO}_{4}, 0.1$
$\mathrm{mM} \mathrm{Na}_{3} \mathrm{P}_{2} \mathrm{O}_{7}$, and $0.1 \mathrm{~mm}$ phenyl methyl sulfonyl fluoride and protease inhibitor), and proteins were denatured and reduced by boiling with Laemmli sample buffer with $0.1 \% \beta$-mercaptoethanol and $10 \mathrm{~mm}$ dithiothreitol. One-fifth of the lysate from each pineal was resolved by SDS-PAGE, transferred onto polyvinylidene fluoride membrane, and immunoblotted with primary antibodies. Specific proteins were visualized with either horseradish peroxidase-conjugated secondary antibodies followed by enhanced chemiluminescence or using the Odyssey infrared imaging system (LI-COR Biosciences). Immunoblots were quantified using ImageJ software, values were normalized to the expression of 14-3-3 and represented graphically as percentage change relative to expression in control animals. Unpaired twotailed $t$ test was calculated for statistical significance.

Immunohistochemistry. Pineals were fixed overnight in $4 \%$ paraformaldehyde and 5\% sucrose PBS, cryoprotected, and embedded and frozen in Tissue-Tek OCT compound. Tissues were cryosectioned at $12 \mu \mathrm{m}$ and mounted onto glass slides. Five sections from each condition were mounted on the same slide to be processed identically. Slides were processed for immunohistochemistry as described previously (Calinescu et al., 2009). Hoechst 3332 was used as nuclear counterstain. Micrograph images were obtained with an Olympus Fluoview 500 confocal microscope using the same parameters for experimental and control samples. Adobe Photoshop CS2 (Adobe Systems) was used to construct the figures and overlays.

Quantitative real-time PCR. Total RNA was extracted from pineals using the RNeasy mini kit. Complementary DNA was generated with the Superscript II first strand synthesis kit using the manufacturer's protocol with oligo(dT). Quantitative real-time PCR (QPCR) analysis was performed using the Stratagene MX3000P cycler and Brilliant II SYBR Green QPCR master mix. Primer sequences are as follows: for $n g f$ (XM_001067130) forward: GGACGCAGCTTTCTATCCTG, reverse: CTGTGTCAAGGGAATGCTGA; for $n t 3$ (NM_031073) forward: CGGATGCCATGGTTACTTCT, reverse: GATATCCGCCTGGATCAACT; for a anat (NM_012818) forward: TGCCACCAGTGCGTTTGAGATTG, reverse: GCCTGTGTAGTGTCAGCGACTCCTG; for hprt (NM_012583) forward: GCAGACTTTGCTTTCCTTGG, reverse CCGCTGTCTTTTAGGCTTTG. Threshold cycles generated by the Stratagene MX3000P software were used to calculate specific mRNA expression with the $\Delta \Delta \mathrm{Ct}$ method, normalizing values to the expression of the housekeeping gene hprt. Results are represented graphically as percentage expression relative to control. Unpaired two-tailed $t$ test was performed for statistical analysis. Specificity of amplification was confirmed by agarose gel electrophoresis of the amplification products, which showed single bands of the expected size.

Isoproterenol treatment. A cohort of rats received a single injection of either vehicle (PBS) or isoproterenol $\mathrm{HCl}$ intraperitoneally at a concentration of $1.4 \mathrm{mg} / \mathrm{kg}$ body weight at ZT6 and was killed $4 \mathrm{~h}$ later at ZT10. A separate cohort of animals, maintained in constant light for $12 \mathrm{~d}$, received 12 consecutive daily injections of either vehicle (PBS) or isoproterenol administered between ZT11-ZT12.

\section{Results}

Silencing of sympathetic activity in the pineal results in a progressive decrease in axonal proteins

Sympathetic activity is tightly associated with the activity of AANAT in the pineal gland (Bowers and Zigmond, 1982). To evaluate the effects of activity deprivation on sympathetic terminal arbors in the pineal, we exposed animals to LL to inhibit SCG-pineal circuit activity. A light pulse at night is well known to block the release of norepinephrine (Drijfhout et al., 1996), which abolishes melatonin release (Drijfhout et al., 1996; Liu and Borjigin, 2005; Huang et al., 2008) and shuts down the production of AANAT mRNA and protein in the pineal gland (Borjigin et al., 1995; Roseboom et al., 1996; Gastel et al., 1998; Huang et al., 2008; Wongchitrat et al., 2009). Thus, the suppression of AANAT or melatonin production by light at night is indicative of the silencing of the SCG-pineal circuit activity, since the two events are coupled extremely tightly (Roseboom et al., 1996). In all anal- 
A
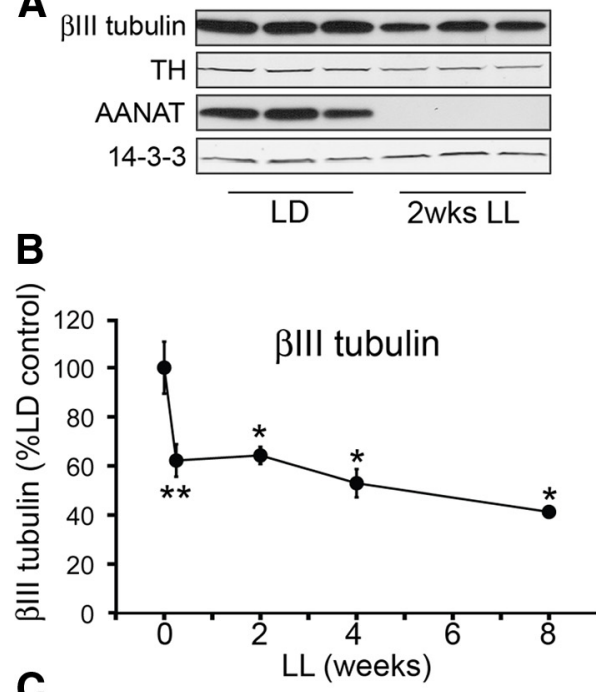
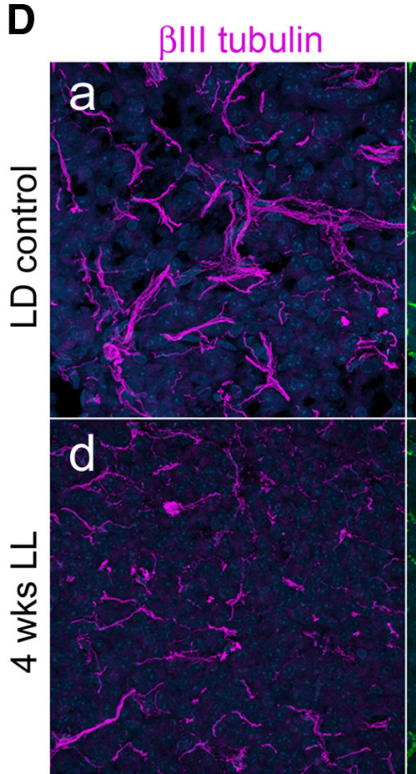

TH

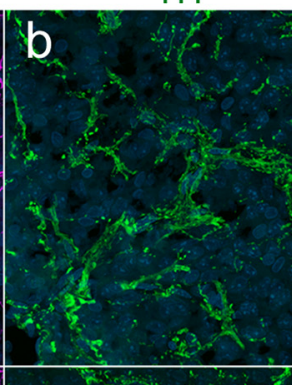

$\beta$ III tubulin/TH

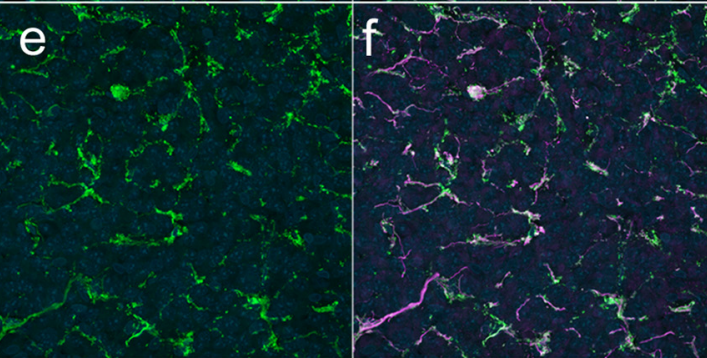

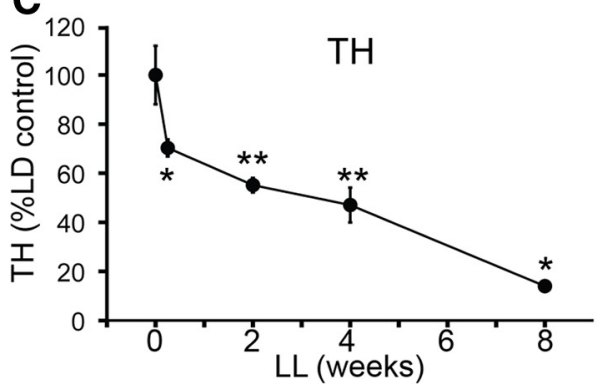

Figure 1. Constant light results in a progressive decrease in expression of axonal proteins in the pineal. $A$, Representative Western blot analysis of pineal lysates from animals reared in either $L D$ conditions or following 2 weeks of LL, probed with antibodies against $\beta$ III tubulin, TH, AANAT, and 14-3-3. In this and all other Western blots, each lane represents pineal lysate from a different animal and expression of 14-3-3 is monitored for quantifying protein levels. $B, \beta$ III tubulin protein levels following light exposures of increasing durations $(0,0.25,2,4$, and 8 weeks). The values are expressed as percentage relative to $L D$ control animals, which are as follows: $62 \%$ at 0.25 weeks $(42 \mathrm{~h} ; n=6), 64 \%$ at 2 weeks $(n=3), 53 \%$ at 4 weeks $(n=3)$, and $41 \%$ at 8 weeks of LL $(n=3)$. C, TH expression following exposure to constant light is $70 \%$ $(n=6)$ after 0.25 weeks, $55 \%(n=3)$ after 2 weeks, $47 \%(n=3)$ after 4 weeks, and $14 \%(n=3)$ after 8 weeks. Two-tailed $t$ test was performed for statistical analysis; ${ }^{*} p<0.05,{ }^{* *} p<0.01$. Error bars represent SEM. $\boldsymbol{D}$, Pineal glands from control animals reared in LD conditions $(\boldsymbol{a}-\boldsymbol{c})$ and after 4 weeks of $L \mathrm{~L}(\boldsymbol{d}-\boldsymbol{f})$ were analyzed by immunohistochemistry for the presence of axonal markers $\beta$ III tubulin $(\boldsymbol{a}, \boldsymbol{c}$, $\boldsymbol{d}, \boldsymbol{f})$ and $\mathrm{TH}(\boldsymbol{b}, \boldsymbol{c}, \boldsymbol{e}, \boldsymbol{f})$. Note colocalization of $\beta \| l \mathrm{l}$ tubulin and TH and marked decrease in the thickness and density of axonal terminal arbors $(\boldsymbol{d}-\boldsymbol{f})$ following exposure to constant light. Hoechst 3332 was used as nuclear counterstain (blue). All animals were killed at ZT18. The findings shown in $\boldsymbol{D}$ were confirmed in two rats. AANAT levels were assayed in all Western blotting experiments to ascertain the silencing of adrenergic activities when light was present at night.

yses, the silencing of sympathetic activity by light at night was confirmed by the absence of AANAT, the key enzyme in melatonin production.

A cohort of rats was exposed to constant light for increasing periods of time $(0.25,2,4$, and 8 weeks) and the levels of axonal proteins in the pineal were analyzed by Western blot. A representative blot taken after 2 weeks of LL is illustrated in Figure $1 \mathrm{~A}$, which reveals a significant decrease in the neuronal microtubule protein $\beta \mathrm{III}$ tubulin and $\mathrm{TH}$, specific markers for sympathetic axonal fibers in the pineal. This decrease occurs most dramatically at $42 \mathrm{~h}(0.25$ week $)$ of $\mathrm{LL}$, and progresses further with longer exposure times (Fig. $1 B, C$ ). Changes in $\beta$ III tubulin and $\mathrm{TH}$ levels correlate throughout the time course of light exposure (Pearson correlation coefficient $=0.9404)$. To investigate morphological changes of axon terminals in the pineal, we analyzed $\beta$ III tubulin and TH in the pineal gland of animals in LD or following 4 weeks of LL. Immunohistochemical analysis showed that sympathetic axon terminals coexpress $\beta \mathrm{III}$ tubulin and $\mathrm{TH}$ and that long-term exposure to constant light results in a reduction in the density of these fibers along with a reduction in fiber and/or fiber-bundle thickness (Fig. 1D). In constant light, the size of the fiber bundles appears to be smaller. Moreover, the distribution of the fibers appears to be more scattered than in control pineal.
Axons in the pineal represent mainly sympathetic axonal arbors originating from the two SCG

While pineal innervation originates mainly from sympathetic postganglionic neurons in the SCGs (Kappers, 1960; Bertler et al., 1964; Bowers et al., 1984; Patrickson and Smith, 1987), there have been reports of parasympathetic innervation of the pineal from the superior salivatory nucleus via the sphenopalatine ganglion (Larsen et al., 1998; Larsen, 1999). To ensure that our quantitative analysis of neuronal proteins following exposure to constant light reflects sympathetic axonal terminals originating from the SCGs, we analyzed $\beta$ III tubulin and TH by Western blot 6,12 , and $36 \mathrm{~h}$ after bilateral surgical removal of the SCGs $(2 \times \mathrm{SCG})$. The levels of $\beta$ III tubulin and TH decrease rapidly as early as $6 \mathrm{~h}$ postsurgery; $36 \mathrm{~h}$ following $2 \times \mathrm{SCG}, \beta$ III tubulin dramatically decreased and TH was not detectable (Fig. 2A). Further, $36 \mathrm{~h}$ after $2 \times$ SCG, the neuronal proteins are undetectable in the body of the pineal by immunohistochemistry (Fig. $2 B$ ).

\section{Expression of neurotrophins in the pineal is strongly decreased} following activity-deprivation

It is generally accepted that survival of adult sympathetic neurons is less dependent on neurotrophic factors compared with neurons in the perinatal and early postnatal stage (Cowen and Gavazzi, 1998). Little is known, however, about physiological factors that reg- 
A

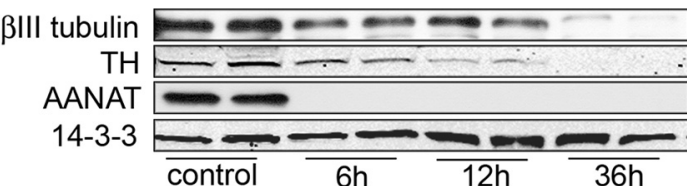

B

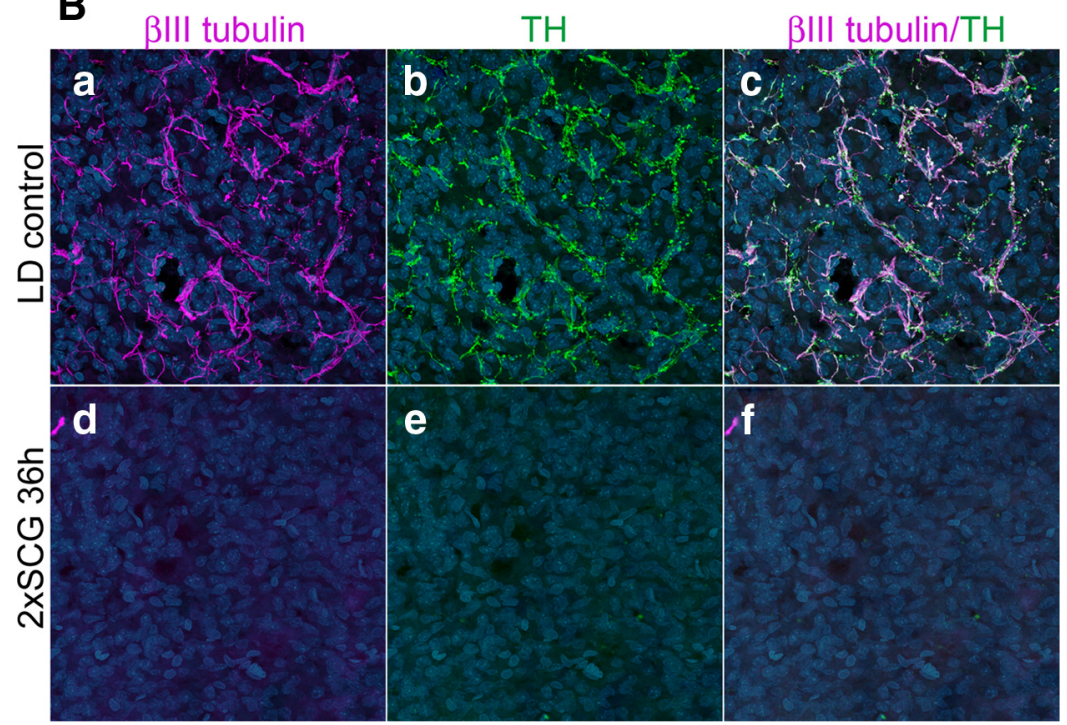

Figure 2. Axonal proteins in the pineal represent mainly sympathetic axonal arbors originating from the two SCGs. $A$, Western blot analysis of $\beta$ III tubulin and TH in pineals from control and ganglionectomized animals ( $2 \times S C G) 6,12$, and 36 h after surgical denervation. Note the progressive decrease in TH ( $64 \%$ of control at $6 \mathrm{~h}, 17 \%$ at $12 \mathrm{~h}$, and $1 \%$ at $36 \mathrm{~h} 2 \times \mathrm{SCG}$ ) and $\beta$ Ill tubulin ( $53 \%$ of control at $6 \mathrm{~h}, 40 \%$ at $12 \mathrm{~h}$, and $3 \%$ at $36 \mathrm{~h}$ postdenervation) proteins following bilateral ganglionectomy. Expression of AANAT, which is induced by norepinephrine release from the SCG axon terminals, was monitored to confirm the surgical denervation. $\boldsymbol{B}$, Pineal glands from control $(\boldsymbol{a}-\boldsymbol{c})$ and $36 \mathrm{~h}$ following $2 \times \mathrm{SCG}(\boldsymbol{d}-\boldsymbol{f})$ were analyzed by immunohistochemistry for the expression of axonal markers $\beta$ III tubulin $(\boldsymbol{a}, \boldsymbol{c}, \boldsymbol{d}, \boldsymbol{f})$ and TH $(\boldsymbol{b}, \boldsymbol{c}, \boldsymbol{e}, \boldsymbol{f})$. Hoechst 3332 was used as nuclear counterstain (blue). Note that $\beta$ III tubulin and TH colocalize in axonal fibers in the control sample $(\boldsymbol{a}-\boldsymbol{c})$ and that, $36 \mathrm{~h}$ following surgical denervation, axonal proteins are undetectable. All animals were killed at ZT15. These results $(\boldsymbol{A}, \boldsymbol{B})$ were confirmed in $>5$ independent experiments.

ulate expression of neurotrophins in targets of sympathetic neurons in adult animals. Since we observed a profound decrease in pineal innervation following activity deprivation, and since there is ample evidence documenting that neurotrophins promote sympathetic axonal growth, we asked whether deprivation of sympathetic input to the pineal results in changes in expression of NGF and NT3, the most abundant neurotrophins expressed in the adult pineal (QPCR; data not shown).

Results (Fig. $3 A, B$ ) show that exposure to constant light results in a profound decrease in proNGF protein $(\sim 34 \mathrm{kDa})$. Western blot quantification of proNGF expression in the pineal during the time course of light exposure shows that there is a significant decrease (50\%) in proNGF expression as early as $42 \mathrm{~h}$ in LL and this decrease is even more severe $(86 \%)$ following 8 weeks of LL treatment (Fig. 3B). The decrease in proNGF expression correlates with the decrease in $\beta$ III tubulin (Pearson = 0.9109 ) and TH (Pearson $=0.9049)$. In the pineal, the most abundant form of NGF is the $34 \mathrm{kDa}$ protein (Bierl et al., 2005; Bierl and Isaacson, 2007; Randolph et al., 2007). The mature NGF $(12.5 \mathrm{kDa})$ is a minor form of NGF and is not detected by Western blot analysis. In our experimental conditions, with very stringent reducing agents in the lysis buffer, the only band we observed in the pineal using the polyclonal antibody $\mathrm{M}-20$ that also detected the mature NGF was the $\sim 34 \mathrm{kDa}$ band (Fig. $3 \mathrm{Ca}$ ). This band was not detected when the antibody was mixed with blocking peptide (Fig. 3Cb).

Our data agrees with other reports showing proNGF as the most abundant form of NGF protein, with little or no mature
NGF detected in the brain and the pineal gland (Fahnestock et al., 2001; Bierl and Isaacson, 2007). The mature form of NGF is the biologically active form of this molecule (Edwards et al., 1988), with numerous reports showing its role in neural survival and neurite growth through binding and activation of TrkA receptors and retrograde signaling (Zweifel et al., 2005; Campenot, 2009). proNGF binds to the $\mathrm{p} 75^{\mathrm{NTR}}$ low-affinity neurotrophin receptor and induces apoptosis (CasacciaBonnefil et al., 1996; Frade et al., 1996; Roux and Barker, 2002). More recent studies suggest that proNGF can also possess neurotrophic activities (Fahnestock et al., 2004; Al-Shawi et al., 2008). Importantly, NGF is released in the extracellular space as proNGF, where it is processed into the mature form and degraded by proteolytic enzymes (Bruno and Cuello, 2006), suggesting that changes in proNGF in vivo could reflect levels of active and mature NGF, particularly when accompanied by changes in $n g f$ mRNA.

To test whether the decrease in NGF protein is reflected at the transcriptional level, we performed real-time QPCR analysis of pineal $n g f$ transcripts. Light exposure resulted in a profound decrease (87\%) in $n g f$ transcripts, which occurred as early as $42 \mathrm{~h}$ in LL. Longer exposure did not produce further decrease (Fig. 3D). We observed a significant decrease in $n t 3$ transcripts as well ( $58 \%$ after $42 \mathrm{~h} \mathrm{LL;} \mathrm{Fig.} 3 \mathrm{E}$ ), although lower in magnitude than the decrease in $n g f$. These results suggest that target expression of $n g f$ and $n t 3$ is regulated by neural activity through the nighttime release of norepinephrine and activation of $\beta$-adrenergic receptors. To test this hypothesis further, we injected animals with isoproterenol (a $\beta$-adrenergic agonist) during the day when sympathetic activity in the pineal is minimal, and analyzed expression of $n g f$ and $n t 3$ mRNA $4 \mathrm{~h}$ later. Results show that expression of $n g f$ and $n t 3$ is increased 5.54-fold and 2.33-fold, respectively (Fig. $3 F$ ), indicating that neurotrophin expression in the pineal is regulated through sympathetic activation. Effectiveness of the isoproterenol injection was confirmed by analyzing the expression of aanat $\mathrm{mRNA}$, which increased $\sim 500$-fold following isoproterenol treatment (data not shown).

Functional activation of the pineal with a $\beta$-adrenergic agonist rescues the light-induced decrease in axonal proteins and proNGF Since we observed that functional silencing of neuronal activity results in a decrease of sympathetic innervation and this correlates with a decrease in NGF expression, and since we can induce $n g f$ mRNA expression in the pineal with isoproterenol, we tested whether we could rescue the expression of axonal proteins in the activity-deprived pineal with $\beta$-adrenergic stimulation. We kept animals in either LD or LL conditions for $12 \mathrm{~d}$ and treated them daily with either vehicle or isoproterenol. The injection was administered intraperitoneally $30 \mathrm{~min}$ before the normal onset of the dark period. Levels of $\beta$ III tubulin, TH, and proNGF are profoundly decreased in animals deprived of sympathetic stimulation, whereas $\beta$-adrenergic stimulation rescues the levels of ax- 
A

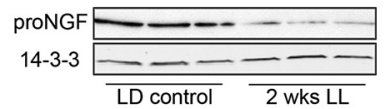

B

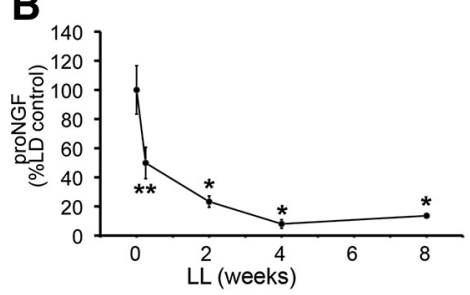

C

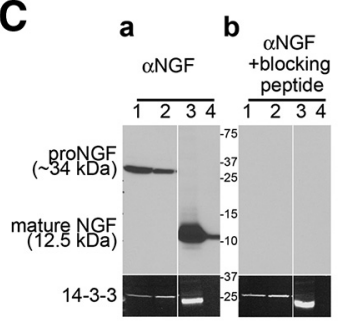

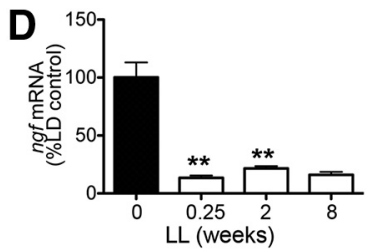

E

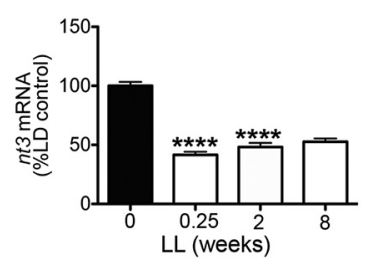

$\mathbf{F}$

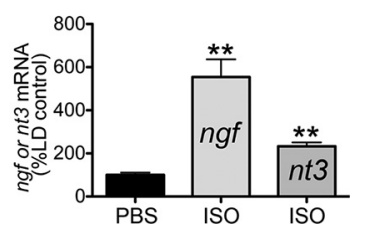

Figure 3. Neurotrophin (NGF and NT3) expression in the pineal is progressively decreased following exposure to constant light. $A$, A representative Western blot analysis of pineal lysates from animals reared in either $L D$ conditions or after 2 weeks of $L L$ shows decrease in expression of proNGF in animals reared in LL. $\boldsymbol{B}$, Time course of changes in proNGF expression following exposure to constant light represented as percentage proNGF relative to $L D$ control: $50 \%$ after 0.25 weeks $\mathrm{LL}(n=6), 23 \%$ after 2 weeks LL $(n=3), 8 \%$ at 4 weeks LL $(n=3)$, and $14 \%$ after 8 weeks $\mathrm{LL}(n=3)$. C, Expression of proNGF in the pineal. Daytime pineal lysates from two independent rats (lanes 1 and 2) were resolved by SDS-PAGE and analyzed by immunoblot with the polyclonal anti-NGF antibody $(\boldsymbol{a})$ or with the same antibody preincubated with blocking peptide $(\boldsymbol{b})$. Two positive controls were used: lysate of male mouse submandibulargland (lane3) and 7 ng of pure NGF (N6009; Sigma) (lane4). Note that the $34 \mathrm{kDa}$ band is the only detectable NGF species in the pineal lysates. Preincubation of the antibody with the NGF blocking peptide results in no NGF signal ( $\boldsymbol{b}$ ), confirming specificity of the pineal $34 \mathrm{kDa}$ band. $\boldsymbol{D}$, Time course of $n g f m R N A$, analyzed by $Q P C R$, in pineal glands of animals maintained in 12:12 $h L D$ conditions $(n=9)$ or following exposure to $L L$, shows a decrease in expression of $n g f: 13 \%$ after 0.25 weeks $\mathrm{LL}(n=4), 21.5 \%$ after 2 weeks $\mathrm{LL}(n=3)$, and $16 \%$ after 8 weeks $\mathrm{LL}(n=2)$. $\boldsymbol{E}$, Time course of $n t 3$ mRNA, analyzed by QPCR, in pineal glands of animals maintained in $L D(n=9)$ or following extended periods of $\mathrm{LL}$, shows a decrease in expression of $n t 3$ following exposure to constant light: $42 \%$ after 0.25 weeks LL $(n=4), 48 \%$ after 2 weeks LL $(n=3)$, and $47 \%$ after 8 weeks LL $(n=2)$. All animals in $\boldsymbol{A}-\boldsymbol{D}$ were killed at ZT18. $\boldsymbol{F}, \beta$-Adrenergic stimulation with isoproterenol (ISO) induces expression of $n g f$ and $n t 3$ mRNA. Expression of $n g f$ and $n t 3$ in the pineal, analyzed by QPCR $4 \mathrm{~h}$ after a single intraperitoneal injection of isoproterenol administered at ZT6, increased 5.5-fold for $n g f$ $(n=4)$ and 2.3-fold for $n t 3$ compared with vehicle-injected (PBS) controls $(n=3)$. Two-tailed $t$ test was performed for statistical analysis; ${ }^{*} p<0.05,{ }^{* *} p<0.01,{ }^{* * * *} p<0.0001$. Error bars represent SEM. Animals were killed at ZT18. Silencing of circuit activity was confirmed by the absence of AANAT protein following light exposure in $\boldsymbol{A}$ and $\boldsymbol{B}$ and by the suppression of a anat mRNA in $\boldsymbol{D}$ and $\boldsymbol{E}$ following light exposure (data not shown).

onal proteins and proNGF in the pineal despite the continued presence of light (Fig. 4). The expression of AANAT was monitored as a control to confirm efficiency of $\beta$-adrenergic activation on pinealocytes. These data strongly suggest that expression of NGF and of axonal proteins in the pineal are under the control of sympathetic activity.

Axonal growth from unlesioned sympathetic neurons is profoundly impaired in the absence of neural activity

Pineal gland displays time-dependent collateral growth of sympathetic fibers following a partial denervation (Dornay et al., 1985; Lingappa and Zigmond, 1987; Kuchel and Zigmond, 1991). Since NGF has been shown to promote collateral sprouting in other systems (Diamond et al., 1987; Doubleday and Robinson, 1994), and

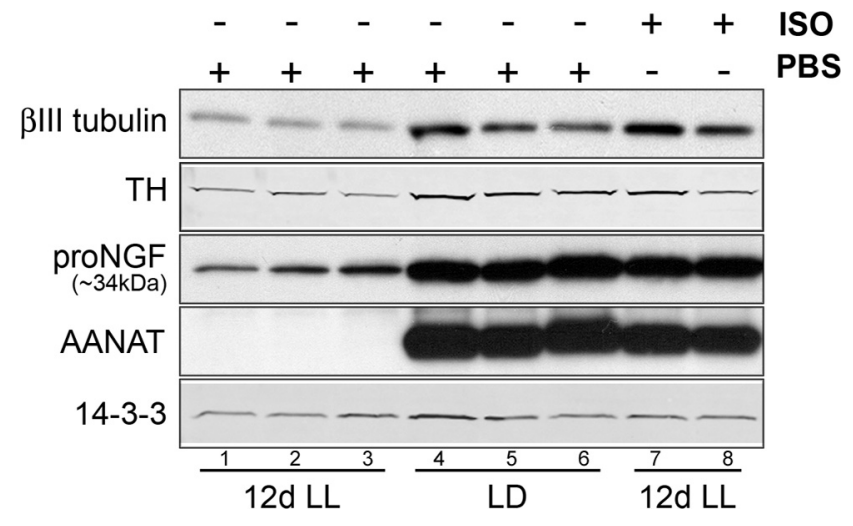

Figure 4. Functional activation of the pineal with a $\beta$-adrenergic agonist rescues the lightinduced decrease in axonal proteins and proNGF. Levels of $\beta$ III tubulin, TH, and proNGF were analyzed by Western blot in control pineal lysates from animals reared in $L D$ conditions $(n=3$; lanes $4-6)$ or maintained for 12 d in constant light ( $n=3$; lanes $1-3,7$, and 8 ) and received 12 consecutive daily injections of vehicle (lanes 1-3) or isoproterenol (ISO; lanes 7 and 8 ) ( $n=3$, two lanes were shown). $\beta I I I$ tubulin, TH, and proNGF are decreased in animals maintained in LL (lanes 1-3) compared with animals reared in LD (lanes 4 -6). This decrease is rescued with 12 daily administrations of isoproterenol (lanes 7and 8). AANAT, which is found only during the dark phase of the light cycle and is induced by the activation of pineal $\beta$-adrenergic receptors, was monitored to confirm the effect of light exposure and efficacy of the isoproterenol injection. All animals were killed at ZT18.

since we observe a decrease in NGF protein and mRNA in the pineals of animals in the absence of sympathetic activities, we tested the consequences of LL on collateral sprouting.

We analyzed the levels of $\beta$ III tubulin, TH, and NGF in the pineal glands of rats following unilateral sympathectomy with or without light exposures of varying durations. Results (Fig. 5) demonstrate a profound consequence of inactivity on collateral growth of sympathetic neurons spared in the partial denervation injury. Following unilateral removal of one SCG, the pineal gland displayed a $50 \%$ loss of sympathetic fibers at $42 \mathrm{~h}$, which is gradually compensated for by the growth of the contralateral SCG neurons spared by the injury following injury (Fig. $5 B, C$, black circles) if animals experience normal sympathetic activity at night in LD. Importantly, the collateral growth of the spared SCG is associated with increased NGF protein expression in the partially injured pineal gland (Fig. 5D, black circles). However, when the injured animals were housed in LL, which blocks the sympathetic activity to the pineal gland, the compensatory collateral growth of SCG neurons is completely suppressed (Fig. $5 B, C$, gray diamonds). Correspondingly, NGF protein (Fig. $5 D$, gray diamonds) and mRNA (data not shown) expression in the injured pineal gland is also dramatically reduced.

\section{Discussion}

Activity is critical for neural plasticity in the adult nervous system. Previous investigations of plasticity relied heavily on denervation injury to reduce neuronal activity. We took advantage of the fact that neural activity in the pineal gland can be controlled simply by light to analyze activity-dependent plasticity in the adult sympathetic nervous system. This maneuver enabled us to monitor the effect of silencing activity in living mammals without the need for invasive procedures, genetic manipulations, or in vitro analysis. We report here that in the absence of neural activity, sympathetic terminal arbors in the pineal gland undergo profound structural changes and a corresponding decrease in structural and functional proteins. The suppression of sympathetic innervation parallels a decrease in neurotrophin expression, which can be restored by $\beta$-adrenergic stimulation. Further, axonal growth of the uninjured SCG neurons, 
A

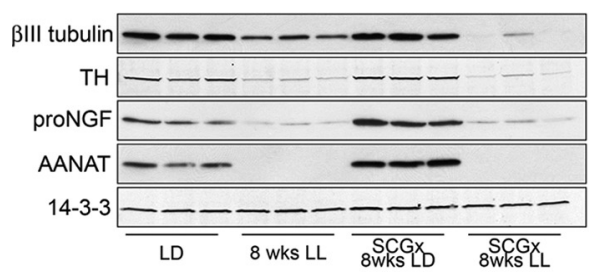

C

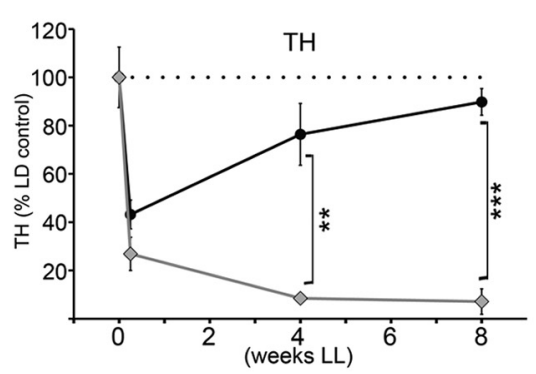

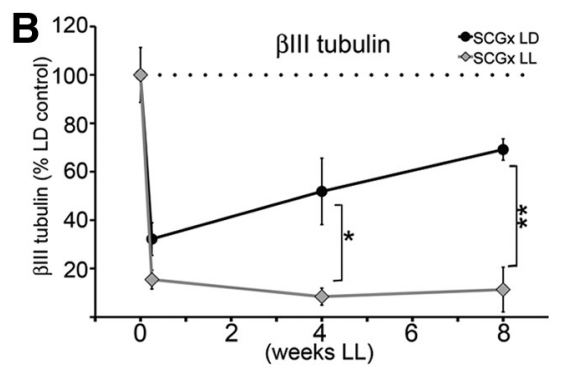

D

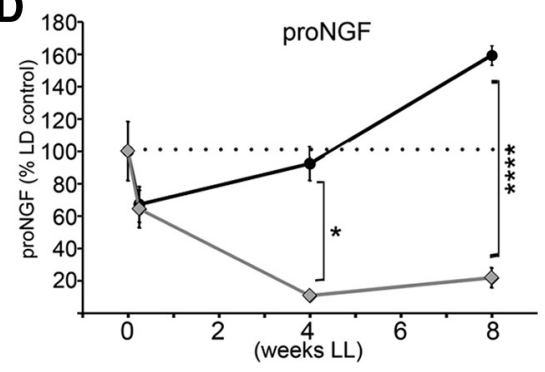

Figure 5. Axonal growth from uninjured SCG neurons following unilateral SCG removal is profoundly impaired in the absence of neural activity. $\boldsymbol{A}$, A representative Western blot of pineal lysates from control animals kept in LD or LL, and from animals which had one SCG surgically removed, and were maintained after surgery for 8 weeks in LD (SCGx 8wks LD) or in LL (SCGx 8wks LL). Membranes were probed with antibodies against $\beta$ III tubulin, TH, NGF, AANAT, and 14-3-3. B, Time course of $\beta$ III tubulin levels in the pineal following unilateral ganglionectomy $(0,0.25,4$, and 8 weeks) from animals reared in either LD (SCGx LD) or LL (SCGx LL). Values are expressed as percentage relative to LD control animals: $32 \%$ at 0.25 weeks ( $42 \mathrm{~h} ; n=6), 52 \%$ at 4 weeks $(n=3)$, and $69 \%$ at 8 weeks $(n=3)$ in the LD samples; and $14 \%$ at 0.25 weeks $(n=6), 8 \%$ at 4 weeks $(n=3)$, and $11 \%$ at 8 weeks $(n=3)$ in animals maintained in $\mathrm{LL}$ conditions. $C$, Time course of TH protein following unilateral ganglionectomy from animals reared in either LD or LL. Values are relative to LD controls: $43 \%$ at 0.25 weeks $(42 \mathrm{~h} ; n=6), 76 \%$ at 4 weeks $(n=3)$, and $90 \%$ at 8 weeks $(n=3)$ in the LD samples; and $27 \%$ at 0.25 weeks $(n=6), 8 \%$ at 4 weeks $(n=3)$, and $7 \%$ at 8 weeks $(n=3)$ in LL samples. D, Time course of proNGF ( $\sim 34 \mathrm{kDa}$ ) expression following unilateral ganglionectomy from animals reared in either LD or LL, expressed as percentage relative to LD control animals: $67 \%$ at 0.25 weeks $(42 \mathrm{~h} ; n=6), 92 \%$ at 4 weeks $(n=3)$, and $159 \%$ at 8 weeks $(n=3)$ in the LD samples; and $64 \%$ at 0.25 weeks $(n=6), 11 \%$ at 4 weeks $(n=3)$, and $22 \%$ at 8 weeks $(n=3)$ in animals maintained in LL conditions. Unpaired, two-tailed $t$ test was performed for statistical analysis comparing the time point values for each protein to the LD control values. The values between the SCGx LD and SCGx LL at each time point: ${ }^{*} p<0.05,{ }^{* *} p<0.01$, ${ }^{* * *} p<0.001,{ }^{* * *} p<0.0001$. Error bars represent SEM. All animals were killed at ZT18. Again, silencing of the sympathetic activity by light was confirmed by the absence of AANAT protein in the pineal gland of rats exposed to light at night. Of note, histological confirmation of sprouting following partial pineal denervation has been previously demonstrated (Lingappa and Zigmond, 1987).

stimulated by unilateral ganglionectomy, is severely impaired by activity deprivation. Our findings support a transsynaptic positive feedback loop between the sympathetic neurons and their target to promote activity-dependent neuroplasticity; neural activity in the SCG promotes postsynaptic function and expression of neurotrophins, which may in turn feed back onto sympathetic neurons to promote and maintain their structural integrity.

Role of neuronal activity in regulating axon structure

Numerous studies have analyzed neuroplasticity in the developing and mature sympathetic nervous system, focusing mainly on the survival and neurite extension of sympathetic neurons following injury or during aging and with or without exogenous neurotrophins (Cowen and Gavazzi, 1998). The effect of physiological neural activity on the structural plasticity of sympathetic axonal terminals is less understood. Our studies demonstrate that silencing neuronal activity dramatically reduces levels of $\mathrm{TH}$, a marker of sympathetic neurons. Activitydependent regulation of TH has been demonstrated in the peripheral nervous system (Zigmond and Chalazonitis, 1979; Zigmond et al., 1985; Rittenhouse et al., 1988; Chevalier et al., 2008). Rats continuously exposed to light for $20 \mathrm{~d}$ display $\sim 50 \%$ decrease in TH mRNA expression in the SCG (Gallara et al., 2004). Our data complement these existing studies and demonstrate that silencing the circuit activity by light results in a marked reduction of sympathetic axon branches in the pineal and a corresponding decrease in TH protein.

One unexpected finding is the rapid regulation by activity of $\beta$ III tubulin, a component of microtubule cytoskeleton widely used as a neuronal marker (Katsetos et al., 2003). $\beta$ III tubulin expression is greatest during development when axon growth and maturation are most active; levels subsequently decrease and then stabilize in the adult nervous system (Jiang and Oblinger, 1992). Dynamic regulation of $\beta$ III tubulin in adult mammals has been reported in injured dorsal root ganglion (Jiang et al., 1994) and following stimulation of cAMP signaling pathway (Gallara et al., 2004; Liu and Brady, 2004). Our results demonstrate for the first time that silencing of neuronal activity leads to a profound decrease in $\beta$ III tubulin in a sympathetic target. Importantly, $\beta$ III tubulin levels decline by one third after only $42 \mathrm{~h}$ of constant light. As far as we know, this is the first report to date of a rapid turnover of structural elements of adult axons. These findings suggest that structural remodeling of axon terminals is a highly dynamic process and that maintenance of integrity of sympathetic axons depends critically on sustained activity of the neural circuit. It remains to be determined whether light at night exerts comparable effects on other peripheral targets controlled by the central circadian clock (Kalsbeek et al., 2006).

Previous reports have shown that sympathetic innervation to a partially denervated pineal gland is restored over time through compensatory growth of neurons in the spared SCG (Dornay et al., 1985; Lingappa and Zigmond, 1987; Kuchel and Zigmond, 1991). This experimental system is therefore suitable to investigate the anatomical correlate of the empiric observation that repeated use of the circuit enhances structural and functional recovery (a guiding principle for rehabilitation of patients with neurological injury). Using light to repress activity of the SCG-pineal circuit, we were able to unambiguously determine that neuronal activity is required for reestablishment of axonal proteins after unilateral denervation of the pineal gland in vivo. These findings provide perhaps the strongest support yet that activity of a circuit is essential for reestablishment and maintenance of anatomical connections following injury. Overall, the studies provide support of the concept that optimal rehabilitation requires activation of the dysfunctional circuit. Further work using this experimental paradigm could clarify the molecular mechanisms of activitydependent compensatory axonal growth and may prove useful in testing interventions to facilitate neural circuit rehabilitation in an in vivo system.

Role of neuronal activity in NGF expression in target tissues Regulation of NGF expression by norepinephrine has been investigated before with mixed results. In animal models of heart dis- 
ease, chronic norepinephrine treatment in vivo leads to an initial increase of NGF, which decreases at later times when congestive heart failure ensues (Qin et al., 2002; Kimura et al., 2010). The early induction of NGF by norepinephrine in the heart is comparable to activation of NGF expression by a single injection of isoproterenol (Fig. 3F). The subsequent decrease of NGF expression in the heart, despite the continuous infusion of norepinephrine, may reflect negative feedback mechanisms in response to the chronic adrenergic stimulation. In contrast to the earlier report showing that decreased activity did not lead to decreased NGF in one of the sympathetic targets (Liu et al., 1996), our studies clearly demonstrate that sympathetic activity is essential for target expression of NGF.

In the pineal gland 3 weeks following bilateral SCG removal, levels of NGF $(\sim 34 \mathrm{kDa})$ increase significantly (Randolph et al., 2007). In contrast, our data show that silencing sympathetic activity by light at night decreases levels of NGF mRNA and protein; conversely, stimulation of sympathetic activity by activation of $\beta$-adrenergic signaling increases expression of NGF in the pineal. Our conflicting results can be explained by major differences in experimental models. Surgical sympathectomy causes not only circuit inactivation, but also results in Wallerian degeneration and activation of phagocytosis. Indeed, increased NGF expression is frequently seen in inflammation and following denervation of target tissues (Weskamp and Otten, 1987; Skaper, 2001). In our study, we were able to completely and noninvasively inhibit activity of the SCG without denervation. Thus, our studies, which use methodology that eliminates surgical stress, denervation, and inflammation, clearly demonstrate sympathetic inactivity lowers NGF in the target tissue.

Because NGF is known to promote collateral sprouting in other systems (Diamond et al., 1987; Doubleday and Robinson, 1994) and was severely suppressed by inactivity of the SCG-pineal circuit (Fig. 3), we examined the combined effects of activity silencing and partial denervation on pineal NGF expression. Our data show that NGF levels are initially decreased in the injured pineal gland but recover in parallel with the compensatory axonal growth. In the presence of constant light, however, the increase in NGF expression is completely suppressed. These studies provide compelling evidence that activity is required for neurotrophin reexpression after injury. The observation that axonal recovery and neurotrophin expression after injury both require activity is consistent with a functional connection between these two events. Furthermore, these studies provide additional support that this model system may be useful in further molecular and pharmacological investigations of activity-dependent (rehabilitative) recovery after injury.

Role of endogenous NGF in retrograde signaling in adult animals While the role of NGF is firmly established for developing nervous systems (Zweifel et al., 2005; Sharma et al., 2010), the NGF function in adult animals is less clear. Administration or deprivation of NGF in vivo alters morphology and survival of mature SCG neurons (Ruit et al., 1990), and in vivo intracerebroventricular infusion of NGF elicits a sprouting response by sympathetic perivascular axons (Isaacson et al., 1992). These studies demonstrate that exogenous NGF influences the remodeling of mature sympathetic axons. Whether the same is true for endogenous NGF is not known.

Our data demonstrate a dramatic suppression of NGF expression when sympathetic activity is shut down by light, and an equally dramatic upregulation of endogenous NGF by adrenergic agonist, both of which are associated with parallel changes in levels of presynaptic axonal marker proteins. When the pineal gland is unilaterally denervated, the spared SCG displays marked compensatory growth, which is associated with high levels of NGF expression in the recovering pineal gland. The arrest of the axonal growth by light is once again associated with the dramatically reduced NGF levels in the pineal gland. These results strongly suggest that NGF production in adult animals is maintained by continued sympathetic activity; which in turn may retrogradely promote the structural integrity of sympathetic axons.

In circuits formed between central neurons or at neuromuscular junctions, a transsynaptic negative feedback mechanism exists to promote homeostatic neuroplasticity (Davis, 2006; Turrigiano, 2007). When central input to the pineal gland was completely shutdown in this study, however, the pineal gland responds with a transsynaptic positive feedback mechanism, likely mediated by NGF, perhaps to conserve resources of the circuit. We conclude that a Hebbian-like response is the dominant mode of neuroplasticity adopted by the sympathetic circuit in the absence of neural activity.

\section{References}

Al-Shawi R, Hafner A, Olsen J, Chun S, Raza S, Thrasivoulou C, Lovestone S, Killick R, Simons P, Cowen T (2008) Neurotoxic and neurotrophic roles of proNGF and the receptor sortilin in the adult and ageing nervous system. Eur J Neurosci 27:2103-2114.

Bertler A, Falck B, Owman C (1964) Studies on 5-hydroxytryptamine stores in pineal gland of rat. Acta Physiol Scand [Suppl] 239:1-18.

Bierl MA, Isaacson LG (2007) Increased NGF proforms in aged sympathetic neurons and their targets. Neurobiol Aging 28:122-134.

Bierl MA, Jones EE, Crutcher KA, Isaacson LG (2005) 'Mature' nerve growth factor is a minor species in most peripheral tissues. Neurosci Lett 380:133-137.

Borjigin J, Wang MM, Snyder SH (1995) Diurnal variation in mRNA encoding serotonin $\mathrm{N}$-acetyltransferase in pineal gland. Nature 378:783-785.

Borjigin J, Li XD, Snyder SH (1999) The pineal gland and melatonin: molecular and pharmacologic regulation. Annu Rev Pharmacol Toxicol 39:53-65.

Bowers CW, Zigmond RE (1982) The influence of the frequency and pattern of sympathetic nerve activity on serotonin $N$-acetyltransferase in the rat pineal gland. J Physiol 330:279-296.

Bowers CW, Dahm LM, Zigmond RE (1984) The number and distribution of sympathetic neurons that innervate the rat pineal gland. Neuroscience 13:87-96.

Brammer M, Binkley S, Mosher K (1982) The rise and fall of pineal $\mathrm{N}$-acetyltransferase in vitro: neural regulation in the developing rat. J Neurobiol 13:487-494.

Bruno MA, Cuello AC (2006) Activity-dependent release of precursor nerve growth factor, conversion to mature nerve growth factor, and its degradation by a protease cascade. Proc Natl Acad Sci U S A 103:6735-6740.

Calinescu AA, Vihtelic TS, Hyde DR, Hitchcock PF (2009) Cellular expression of midkine-a and midkine-b during retinal development and photoreceptor regeneration in zebrafish. J Comp Neurol 514:1-10.

Campenot RB (1977) Local control of neurite development by nerve growth factor. Proc Natl Acad Sci U S A 74:4516-4519.

Campenot RB (2009) NGF uptake and retrograde signaling mechanisms in sympathetic neurons in compartmented cultures. Results Probl Cell Differ 48:141-158.

Casaccia-Bonnefil P, Carter BD, Dobrowsky RT, Chao MV (1996) Death of oligodendrocytes mediated by the interaction of nerve growth factor with its receptor p75. Nature 383:716-719.

Chevalier J, Derkinderen P, Gomes P, Thinard R, Naveilhan P, Vanden Berghe P, Neunlist M (2008) Activity-dependent regulation of tyrosine hydroxylase expression in the enteric nervous system. J Physiol 586:1963-1975.

Cowen T, Gavazzi I (1998) Plasticity in adult and ageing sympathetic neurons. Prog Neurobiol 54:249-288.

Davis GW (2006) Homeostatic control of neural activity: from phenomenology to molecular design. Annu Rev Neurosci 29:307-323. 
Diamond J, Coughlin M, Macintyre L, Holmes M, Visheau B (1987) Evidence that endogenous beta nerve growth factor is responsible for the collateral sprouting, but not the regeneration, of nociceptive axons in adult rats. Proc Natl Acad Sci U S A 84:6596-6600.

Dornay M, Gilad VH, Gilad GM (1985) Compensatory changes in contralateral sympathetic neurons of the superior cervical ganglion and in their terminals in the pineal gland following unilateral ganglionectomy. J Neurosci 5:1522-1526.

Doubleday B, Robinson PP (1994) Nerve growth factor depletion reduces collateral sprouting of cutaneous mechanoreceptive and tooth-pulp axons in ferrets. J Physiol 481:709-718.

Drijfhout WJ, van der Linde AG, de Vries JB, Grol CJ, Westerink BH (1996) Microdialysis reveals dynamics of coupling between noradrenaline release and melatonin secretion in conscious rats. Neurosci Lett 202:185-188.

Edwards RH, Selby MJ, Garcia PD, Rutter WJ (1988) Processing of the native nerve growth factor precursor to form biologically active nerve growth factor. J Biol Chem 263:6810-6815.

Fahnestock M, Michalski B, Xu B, Coughlin MD (2001) The precursor pronerve growth factor is the predominant form of nerve growth factor in brain and is increased in Alzheimer's disease. Mol Cell Neurosci 18:210-220.

Fahnestock M, Yu G, Coughlin MD (2004) ProNGF: a neurotrophic or an apoptotic molecule? Prog Brain Res 146:101-110.

Frade JM, Rodríguez-Tébar A, Barde YA (1996) Induction of cell death by endogenous nerve growth factor through its p75 receptor. Nature 383:166-168.

Gallara RV, Bellavia SL, Serova LL, Sabban EL (2004) Environmental light conditions alter gene expression of rat catecholamine biosynthetic enzymes and Neuropeptide Y: differential effect in superior cervical ganglia and adrenal gland. Brain Res Mol Brain Res 124:152-158.

Gastel JA, Roseboom PH, Rinaldi PA, Weller JL, Klein DC (1998) Melatonin production: proteasomal proteolysis in serotonin $N$-acetyltransferase regulation. Science 279:1358-1360.

Hamburger V (1993) The history of the discovery of the nerve growth factor. J Neurobiol 24:893-897.

Huang Z, Deng J, Borjigin J (2005) A novel H28Y mutation in LEC rats leads to decreased NAT protein stability in vivo and in vitro. J Pineal Res 39:84-90.

Huang Z, Liu T, Chattoraj A, Ahmed S, Wang MM, Deng J, Sun X, Borjigin J (2008) Posttranslational regulation of TPH1 is responsible for the nightly surge of 5-HT output in the rat pineal gland. J Pineal Res 45:506-514.

Huang Z, Liu T, Borjigin J (2010) N-terminal residues regulate proteasomal degradation of AANAT. J Pineal Res 48:290-296.

Isaacson LG, Saffran BN, Crutcher KA (1992) Nerve growth factor-induced sprouting of mature, uninjured sympathetic axons. J Comp Neurol 326:327-336.

Jiang YQ, Oblinger MM (1992) Differential regulation of beta III and other tubulin genes during peripheral and central neuron development. J Cell Sci 103:643-651.

Jiang YQ, Pickett J, Oblinger MM (1994) Comparison of changes in betatubulin and NF gene expression in rat DRG neurons under regenerationpermissive and regeneration-prohibitive conditions. Brain Res 637:233-241.

Kalsbeek A, Palm IF, La Fleur SE, Scheer FA, Perreau-Lenz S, Ruiter M, Kreier F, Cailotto C, Buijs RM (2006) SCN outputs and the hypothalamic balance of life. J Biol Rhythms 21:458-469.

Kappers JA (1960) The development, topographical relations and innervation of the epiphysis cerebri in the albino rat. Zeitschrift Fur Zellforschung Und Mikroskopische Anatomie 52:163-215.

Katsetos CD, Legido A, Perentes E, Mörk SJ (2003) Class III beta-tubulin isotype: a key cytoskeletal protein at the crossroads of developmental neurobiology and tumor neuropathology. J Child Neurol 18:851-866, discussion 867.

Kimura K, Kanazawa H, Ieda M, Kawaguchi-Manabe H, Miyake Y, Yagi T, Arai T, Sano M, Fukuda K (2010) Norepinephrine-induced nerve growth factor depletion causes cardiac sympathetic denervation in severe heart failure. Auton Neurosci 156:27-35.

Klein DC, Coon SL, Roseboom PH, Weller JL, Bernard M, Gastel JA, Zatz M, Iuvone PM, Rodriguez IR, Bégay V, Falcón J, Cahill GM, Cassone VM, Baler R (1997) The melatonin rhythm-generating enzyme: molecular regulation of serotonin $N$-acetyltransferase in the pineal gland. Recent Prog Horm Res 52:307-357, discussion 357-358.

Korsching S, Thoenen H (1983) Nerve growth factor in sympathetic ganglia and corresponding target organs of the rat: correlation with density of sympathetic innervation. Proc Natl Acad Sci U S A 80:3513-3516.
Kuchel GA, Zigmond RE (1991) Functional recovery and collateral neuronal sprouting examined in young and aged rats following a partial neural lesion. Brain Res 540:195-203.

Larsen PJ (1999) Tracing autonomic innervation of the rat pineal gland using viral transneuronal tracing. Microsc Res Tech 46:296-304.

Larsen PJ, Enquist LW, Card JP (1998) Characterization of the multisynaptic neuronal control of the rat pineal gland using viral transneuronal tracing. Eur J Neurosci 10:128-145.

Levi-Montalcini R, Angeletti PU (1968) Nerve growth factor. Physiol Rev 48:534-569.

Lingappa JR, Zigmond RE (1987) A histochemical study of the adrenergic innervation of the rat pineal gland: evidence for overlap of the innervation from the two superior cervical ganglia and for sprouting following unilateral denervation. Neuroscience 21:893-902.

Liu DT, Reid MT, Bridges DC, Rush RA (1996) Denervation, but not decentralization, reduces nerve growth factor content of the mesenteric artery. J Neurochem 66:2295-2299.

Liu HH, Brady ST (2004) cAMP, tubulin, axonal transport, and regeneration. Exp Neurol 189:199-203.

Liu T, Borjigin J (2005) Reentrainment of the circadian pacemaker through three distinct stages. J Biol Rhythms 20:441-450.

Lockhart ST, Turrigiano GG, Birren SJ (1997) Nerve growth factor modulates synaptic transmission between sympathetic neurons and cardiac myocytes. J Neurosci 17:9573-9582.

Nelson SB, Turrigiano GG (2008) Strength through diversity. Neuron 60:477-482.

Patrickson JW, Smith TE (1987) Innervation of the pineal gland in the rat: an HRP study. Exp Neurol 95:207-215.

Paulsen O, Sejnowski TJ (2000) Natural patterns of activity and long-term synaptic plasticity. Curr Opin Neurobiol 10:172-179.

Qin F, Vulapalli RS, Stevens SY, Liang CS (2002) Loss of cardiac sympathetic neurotransmitters in heart failure and NE infusion is associated with reduced NGF. Am J Physiol Heart Circ Physiol 282:H363-H371.

Randolph CL, Bierl MA, Isaacson LG (2007) Regulation of NGF and NT-3 protein expression in peripheral targets by sympathetic input. Brain Res 1144:59-69.

Rittenhouse AR, Schwarzschild MA, Zigmond RE (1988) Both synaptic and antidromic stimulation of neurons in the rat superior cervical ganglion acutely increase tyrosine hydroxylase activity. Neuroscience 25:207-215.

Roseboom PH, Coon SL, Baler R, McCune SK, Weller JL, Klein DC (1996) Melatonin synthesis: analysis of the more than 150-fold nocturnal increase in serotonin $\mathrm{N}$-acetyltransferase messenger ribonucleic acid in the rat pineal gland. Endocrinology 137:3033-3045.

Roux PP, Barker PA (2002) Neurotrophin signaling through the p75 neurotrophin receptor. Prog Neurobiol 67:203-233.

Ruit KG, Osborne PA, Schmidt RE, Johnson EM Jr, Snider WD (1990) Nerve growth factor regulates sympathetic ganglion cell morphology and survival in the adult mouse. J Neurosci 10:2412-2419.

Sharma N, Deppmann CD, Harrington AW, St Hillaire C, Chen ZY, Lee FS, Ginty DD (2010) Long-distance control of synapse assembly by targetderived NGF. Neuron 67:422-434.

Skaper SD (2001) Nerve growth factor: a neurokine orchestrating neuroimmune-endocrine functions. Mol Neurobiol 24:183-199.

Turrigiano G (2007) Homeostatic signaling: the positive side of negative feedback. Curr Opin Neurobiol 17:318-324.

Weskamp G, Otten U (1987) An enzyme-linked immunoassay for nerve growth factor (NGF): a tool for studying regulatory mechanisms involved in NGF production in brain and in peripheral tissues. J Neurochem 48:1779-1786.

Wongchitrat P, Felder-Schmittbuhl MP, Phansuwan-Pujito P, Pévet P, Simonneaux V (2009) Endogenous rhythmicity of Bmall and Rev-erb alpha in the hamster pineal gland is not driven by norepinephrine. Eur J Neurosci 29:2009-2016.

Zigmond RE, Chalazonitis A (1979) Long-term effects of preganglionic nerve stimulation on tyrosine hydroxylase activity in the rat superior cervical ganglion. Brain Res 164:137-152.

Zigmond RE, Baldwin C, Bowers CW (1985) Rapid recovery of pineal function after partial denervation: a possible role for heteroneuronal uptake of transmitter in modulating synaptic efficacy. J Neurosci 5:142-150.

Zweifel LS, Kuruvilla R, Ginty DD (2005) Functions and mechanisms of retrograde neurotrophin signalling. Nat Rev Neurosci 6:615-625. 